\title{
Simulation of Manipulator as a Part of Space Robotic SYSTEM WEIGHTLESSNESS DEVICE
}

\author{
Sergey Isaenko, Oleg Sochivko, Igor Shardyko, Viktor Titov \\ Russian State Scientific Center for Robotics and Technical Cybernetics, Saint-Petersburg, Russia
}

\begin{abstract}
This paper presents one of approaches to inertia and weight compensation of manipulator, based on data from angular position sensors in the joints and on a-priori known mass-dimensional characteristics of the links. The problem of inertia and weight compensation of manipulator is very old, but it is currently important again [1,2]. Step forward is solving of the weightlessness problem not only for manipulator, but also for the load with variable center of mass position. Since the work objective is to create for a solid body conductions close to zero-gravity, it is also necessary to compensate manipulator's inertia with no load inertial forces modifying. In current work the solid body model is used for the load, however, for more complicated models (material body, physical object) it is impossible to achieve conditions somehow close to zero-gravity conditions by such way. Even so development of this stand is considered to be promising because other methods of ground-based finalization of mobile robotic systems are unknown.
\end{abstract}

Keywords: robotics; manipulation system; simulation; weightlessness device; modelling; immitation
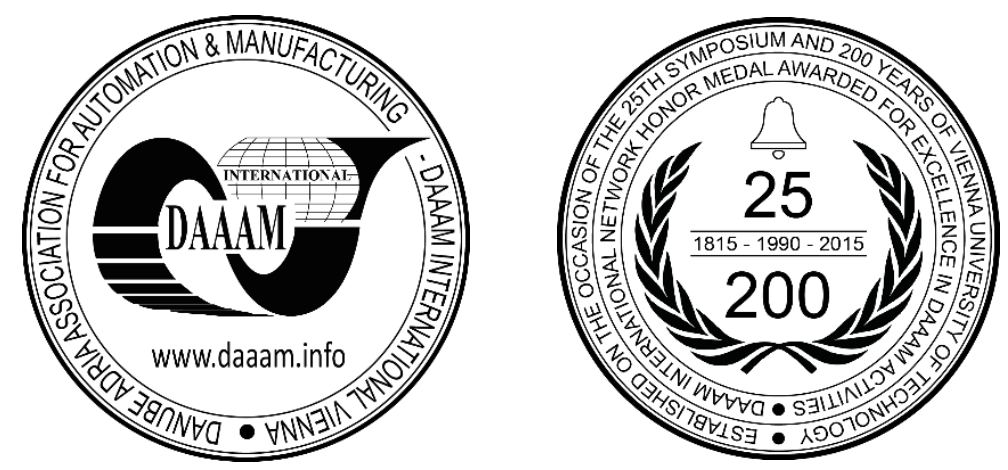

This Publication has to be referred as: Isaenko, S[ergei]; Sochivko, O[leg]; Shardyko, I[gor] \& Titov, V[iktor] (2016). Simulation of Manipulator as a Part of Space Robotic System Weightlessness Device, Proceedings of the 26th DAAAM International Symposium, pp.0554-0561, B. Katalinic (Ed.), Published by DAAAM International, ISBN 978-3-90273407-5, ISSN 1726-9679, Vienna, Austria

DOI: $10.2507 / 26$ th.daaam.proceedings.076 


\section{Introduction}

In future, outer space operations will be implemented predominantly by robots that will reduce human extravehicular activity. Actually, space robotic systems are being developed in many countries, including Russia.

For development tests of space robotic systems on Earth special test benches which enable operation of the units, designed for work in zero-gravity state are required. Such test benches is used for a long time and there are many approaches to their design. For example, there are aquatic weightlessness test benches, flat test benches [3], based on air castors, rope unloading systems etc. An important point is that, none of listed can replicate weightlessness conditions specific for space environment. Each bench is designed for specified problem and isn't suitable for others.

These days new course of space-borne robotic systems design is outlined: mobile robotic system designed for extravehicular activity. Therefore, it requires the development of specialized weightlessness device. Aquatic weightlessness test bench would be suitable for that purpose, but its design is related to a number of technical issues: it's necessary to maintain technical pool and every system modernization implies creation of new model for underwater work, which have neutral buoyancy and is leak-proof. Analysis of upcoming robotic systems in that domain shows that robotic arms joints will have excessive torque capabilities i.e. capable to hold arms even in the earth gravity filed.

For that kind of robotic system a test bench was engineered. It unloads robotic arm joints with manipulating industrial robot. The task of current paper is reasoning of core methods of test bench work algorithms. For achievement of the result uses equation of motion and methods of analytical mechanics. Also some simplifications are included in model.

\section{Weightlessness device description}

The purpose of developed space robotic system weightlessness test bench is servoing of robotic system during different operations. Test bench consists of:

- Weightlessness object

- Space station surface dummy

- Manipulator

- Six-component force and torque sensor

- Software

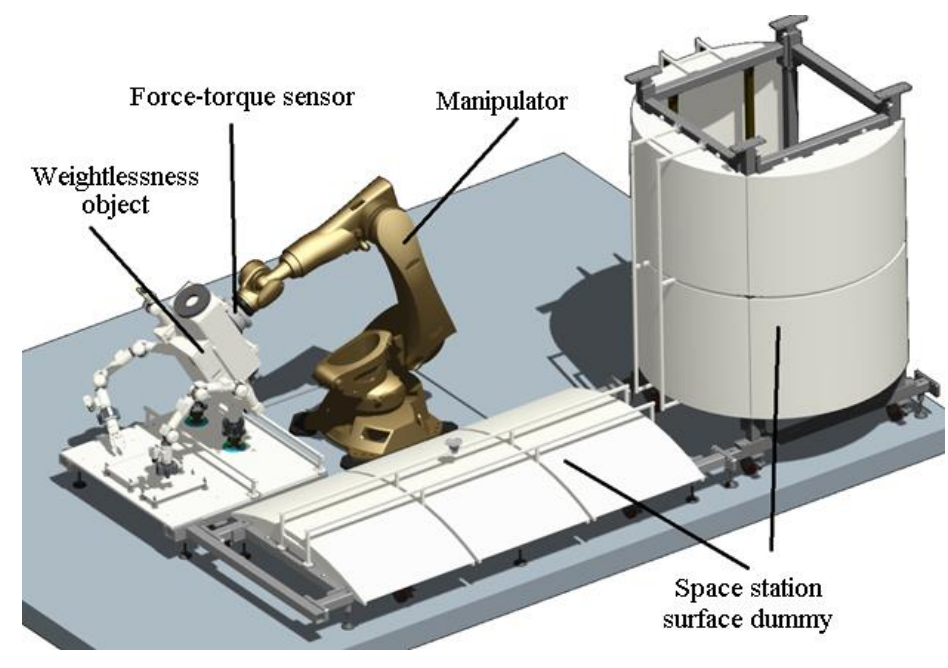

Fig. 1. Test bench general view

Space transport and manipulation system (STMS) is a robotic system designed for extravehicular activity [4-7]. For STMS operations elaboration it is necessary to design a weightlessness test bench for robotic system. From many types of weightlessness test benches the one with hold of the base unit by manipulator arm was chosen for proposed system. Internal tensions are not compensated because of moving parts of STMS are not connected to weightlessness manipulator arm. To enable such system joints must have excessive torque capabilities and possibility to overcome arm segments weight. Preliminary computation shows that drive, capable to derive $70 \mathrm{~N} \cdot \mathrm{m}$ is enough for primary STMS joint.

The test bench purpose is to make STMS velocity characteristics equal to gravity free operation characteristics. Comparing with gravity free operation, during operation on the test bench the joints derive higher torque rates, but STMS has same position and velocity relative to space station dummy, which is a part of test bench.

Control system purpose is to provide conditions, which are approximate to gravity free as much as possible.

Required movement parameters formation is the test bench control system aim. Now and further manipulator arm we mean weightlessness test bench arm, which is connected to STMS by six-component force and torque sensor. 


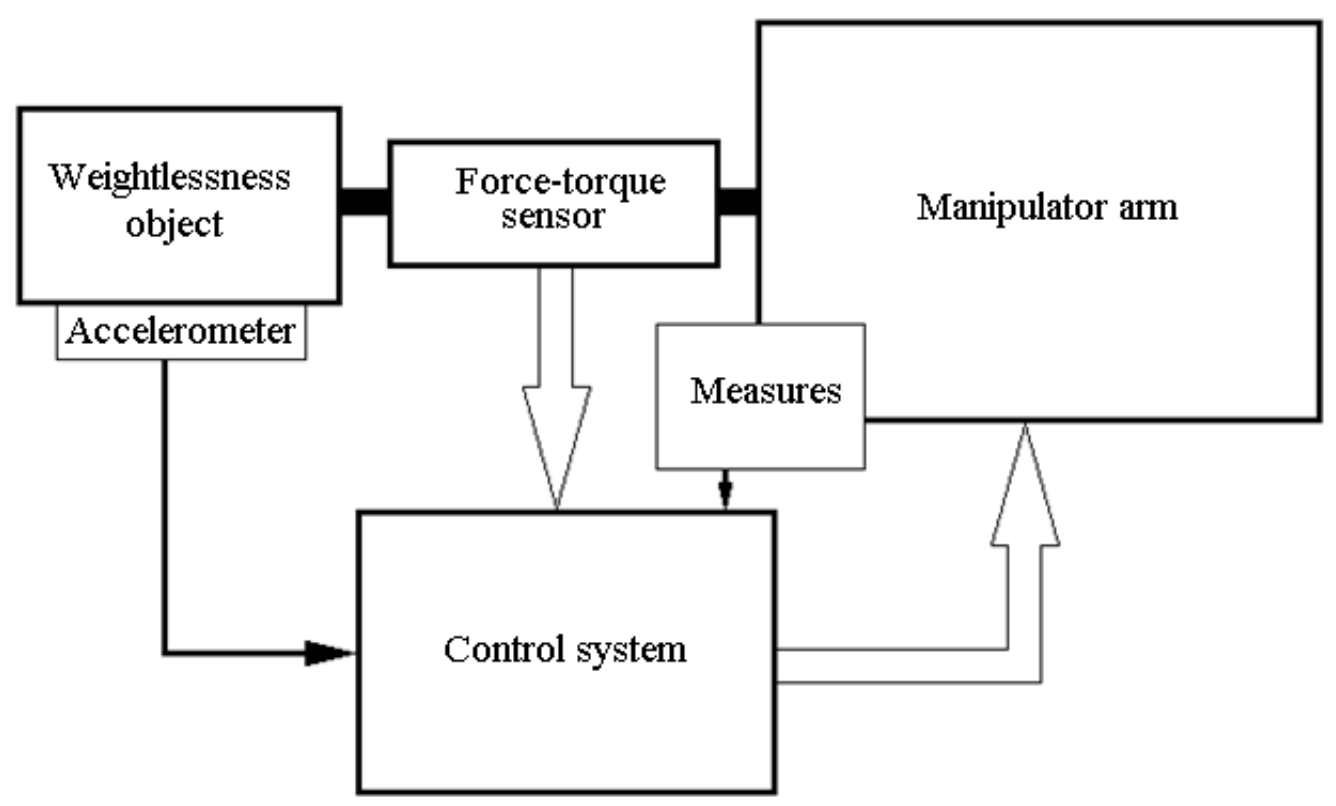

Fig. 2. Test bench function block diagram

Figure 2 shows control system elements and data streams between them.

Force and torque sensor is a device, which measures major vectors of torque and force, projecting them on sensor axes. Output data of torque sensor are measured values of three projections of force and three projections of torque.

Manipulator arm is a device, which is capable to move load in random place and orientate load object, limited with arm working area and estimated velocity characteristics. The minimal number of degrees of freedom to match that demands are six. Moreover, particular characteristics are required - manipulator arm drives must be capable to compensate arm inertia in specified velocity range to make STMS moving characteristics undistorted. Furthermore, arm joints must have velocity control mode, despite the fact that most actual manipulator arms are controlled in position and trajectory modes.

Test bench control system is software which implements operation based on torque and force sensor values.

The concept of test bench is to move object in specified area same way it will move in weightlessness. In weightlessness, by force impact an object should start movement according to force direction until force effect changes or, in case of using test bench, until reaching arm working area limits.

For better performance in weightlessness simulation accelerometer is included in the control system. The accelerometer is attached to an operated object. Acceleration values are considered to make amendments in driving process.

Actually, mathematical model and basic control programs of such test bench are being elaborated. To control with six degrees of freedom manipulator arm and with beforehand unknown characteristics is a sophisticated complex challenge. The main idea of the test bench is to make impossible for STMS to detect that manipulator arm holds it. In other words, STMS movements should be equal to weightlessness operation and holding arm should move STMS in specified position with velocities control, without any preliminary information about position and velocities.

Head-on solution of this task is inaccessible, so at the current stage of development some simplifications are included in model. Later some of them will be rejected. Evolutionally, test bench model will become an appropriate model for wide range of experiments. Some restrictions are included in the current model.

- $\quad$ STMS is considered as an object with fixed center of mass.

- Manipulator arm with six degrees of freedom is simplified to the arm with three degrees of freedom with twodimensional working area.

- Arm joints are considered as high-controllable in torque mode (transition to speed control mode will be implemented later).

- Force and torque sensor is not used in the model because of joints direct torque control.

- Sensors are considered as ideal and inertialess.

In future, listed simplifications will be removed one after another with model complicating and closer approximation to real weightlessness test bench as a result.

In this paper plane motion of object in simulated weightless is covered. The principal issues are defined on the stage of plane motion modelling for arm with 3 degrees of freedom. Transition to six degrees of freedom manipulator arm solid simulation will be realized later. Viable decision for modeling is simplified preliminary solution, because mathematical control apparatus for the arm with 6 degrees of freedom is quite awkward. 


\section{Modelling description}

In this research paper modeling of the manipulator arm with three degrees of freedom as a part of weightlessness test bench for technological ensembles is represented. Weightlessness of operated object is provided by the manipulator arm with three degrees of freedom with parallel joint axes, what is shown in figure 3. Thus, spatial task of manipulator arm with six degrees of freedom comes to plane task.

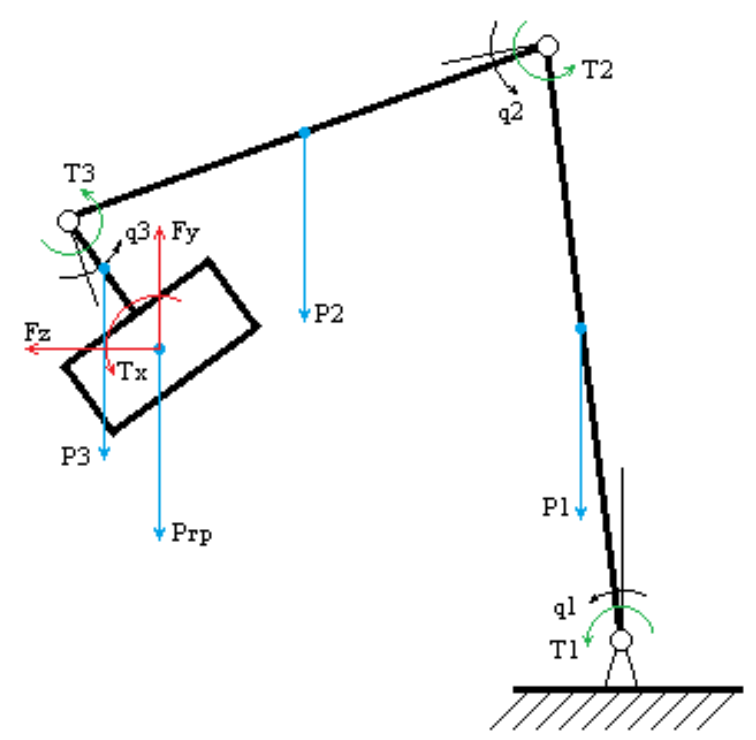

Fig. 3. Diagram of the manipulator with 3 degrees of freedom

Figure 3 keys:

- $\quad \mathrm{q} 1, \mathrm{q} 2, \mathrm{q} 3$ - generalized rotation coordinates of corresponding joints;

- $\quad$ P1, P2, P3, Pload - segments and load weight;

- $\quad \mathrm{T} 1, \mathrm{~T} 2, \mathrm{~T} 3-$ joints torque;

- $\quad \mathrm{Tx}, \mathrm{Fy}, \mathrm{Fz}-$ external torque and forces applied to object centre of mass.

In plane problem case six external force and torque impacts reduce to three: $\mathrm{Y}$ axis force, $\mathrm{X}$ axis force, torque about $\mathrm{X}$ axis. These impacts are active and are applied to the operated object.

Static equations for the manipulator arm with three degrees of freedom, represented on figure 1, are:

$$
\left\{\begin{array}{c}
M_{3}-P \cdot\left(r_{3}+l\right) \cdot \sin \left(q_{1}+q_{2}+q_{3}\right)-P_{3} \cdot\left(\frac{r_{3}}{2}\right) \cdot \sin \left(q_{1}+q_{2}+q_{3}\right)=0 \\
M_{2}-M_{3}+P_{2} \cdot\left(\frac{r_{2}}{2}\right) \cdot \cos \left(q_{1}+q_{2}\right)-R_{y 3} \cdot r_{2} \cdot \cos \left(q_{1}+q_{2}\right)-R_{z 3} \cdot r_{2} \cdot \cos \left(q_{1}+q_{2}\right)=0 \\
M_{1}-M_{2}+P_{1} \cdot\left(\frac{r_{1}}{2}\right) \cdot \sin \left(q_{1}\right)-R_{y 2} \cdot r_{1} \cdot \cos \left(q_{1}\right)-R_{z 2} \cdot r_{1} \cdot \cos \left(q_{1}\right)=0
\end{array}\right.
$$

where:

$\mathrm{M} 1, \mathrm{M} 2, \mathrm{M} 3$ - joints torque;

$\mathrm{P}$ - load weight;

1 - distance between arm ending segment and load mass center;

P1, P2, P3 - arm segments weight;

$\mathrm{r} 1, \mathrm{r} 2, \mathrm{r} 3$ - arm segments length (distance between joints);

$\mathrm{Ry} 3, \mathrm{Rz} 3, \mathrm{Ry} 2, \mathrm{Rz} 2,-$ second and third joints reaction forces $\mathrm{Y}$ and $\mathrm{Z}$ axes projections.

Any interaction of object with environment is interpreted as external effect whether it is intentional or unintentional human applies force impact, or STMS is making efforts to move using own arm joints.

Let's consider the task of the model forming with compensation of forces caused by movement of the manipulator joints. For every separated object equations turn into: 


$$
\left\{\begin{array}{c}
m_{i} \cdot \overline{a_{c l}}=m_{i} \cdot \bar{g}+\overline{V_{l}^{e}}+\overline{R_{\iota}}-\overline{R_{l+1}} \\
\overline{\overline{I_{l}}} \cdot \overline{\varepsilon_{l}}+\overline{\omega_{\iota}} \times\left(\overline{\overline{I_{l}}} \cdot \overline{\omega_{l}}\right)=\overline{M_{c l}^{e}}+\overline{M_{\iota}}-\overline{M_{\iota+1}}+\overline{\rho_{\iota}} \times \overline{R_{\iota}}-\overline{\rho_{l+1}} \times \overline{R_{\iota+1}}
\end{array}\right.
$$

where:

mi - i's number object mass;

aci - i's number object center of mass acceleration

$\mathrm{g}$ - acceleration of gravity

Vie - main vector of external forces applied to i's number object

$\mathrm{Ri}$ - i's number joint reaction;

$\mathrm{Ri}+1-(\mathrm{i}+1)$ 's number joint reaction;

Ii - i's number object inertia tensor;

$\varepsilon i-i$ 's number object angular acceleration;

$\omega \mathrm{i}-\mathrm{i}$ 's number object angular velocity;

Mcie - main vector of external forces applied to i's number object;

$\mathrm{Mi}-\mathrm{i}$ 's number joint torque;

$\mathrm{Mi}+1-(\mathrm{i}+1)$ 's number joint torque;

$\rho i-i$ 's number joint and center of mass connecting vector;

$\rho \mathrm{i}+1-(\mathrm{i}+1)$ 's number joint and center of mass connecting vector.

An important point is that it is necessary to take into account only arm manipulator inertia. Operated object inertia shouldn't be compensated.

For load moving with own inertia compensation torque equations for three joints appear to be:

$$
\left\{\begin{array}{l}
\overline{M_{3}}=\overline{\overline{I_{3}}} \cdot \overline{\varepsilon_{3}}+\overline{\omega_{3}} \times\left(\overline{\overline{I_{3}}} \cdot \overline{\omega_{3}}\right)-\overline{\rho_{\text {гр }}} \times\left(m_{\text {гр }} \cdot \bar{g}\right)-\overline{\rho_{c 3}} \times\left(m_{3} \cdot \bar{g}\right) \\
\overline{M_{2}}=\overline{\overline{I_{2}}} \cdot \overline{\varepsilon_{2}}+\overline{\omega_{2}} \times\left(\overline{\overline{I_{2}}} \cdot \overline{\omega_{2}}\right)+\overline{M_{3}}-\overline{\rho_{c 2}} \times\left(m_{2} \cdot \bar{g}\right)-\overline{\rho_{3}} \times \overline{R_{3}} \\
\overline{M_{1}}=\overline{\overline{I_{1}}} \cdot \overline{\varepsilon_{1}}+\overline{\omega_{1}} \times\left(\overline{\overline{I_{1}}} \cdot \overline{\omega_{1}}\right)+\overline{M_{2}}-\overline{\rho_{c 1}} \times\left(m_{1} \cdot \bar{g}\right)-\overline{\rho_{2}} \times \overline{R_{2}}
\end{array}\right.
$$

where:

M1, M2, M3 are torque values for joints.

I1, I2, I3 -manipulator arm inertia tensors related to joint axis;

$\varepsilon 1, \varepsilon 2, \varepsilon 3$ - manipulator arm angular accelerations;

$\omega 1, \omega 2, \omega 3$ - manipulator arm angular velocities;

$\rho_{\text {load }}-$ third segment and center of mass connecting vector;

$\mathrm{m} 1, \mathrm{~m} 2, \mathrm{~m} 3$, мгр - manipulator arm segments weight and load weight;

$\mathrm{g}$ - gravity force vector;

$\rho c 1, \rho c 2, \rho c 3$ - vectors connecting joint $1(2,3)$ and corresponding segments center of mass;

$\mathrm{R} 2, \mathrm{R} 3$ - second and third reaction forces vectors.

Reaction forces vectors equations are:

$$
\left\{\begin{array}{c}
\overline{R_{3}}=m_{3} \cdot \overline{a_{3}}-m_{3} \cdot \bar{g}-m_{\text {гр }} \cdot \bar{g} \\
\overline{R_{2}}=m_{2} \cdot \overline{a_{2}}-m_{2} \cdot \bar{g}+\overline{R_{3}}
\end{array}\right.
$$

Where

a2, a3 - second and third arm segments accelerations.

All equations in (3) and (4) have vectorial form, consequently, transition from three degrees of freedom arm to six degrees of freedom is possible to accomplish with addition of three generic equations. 


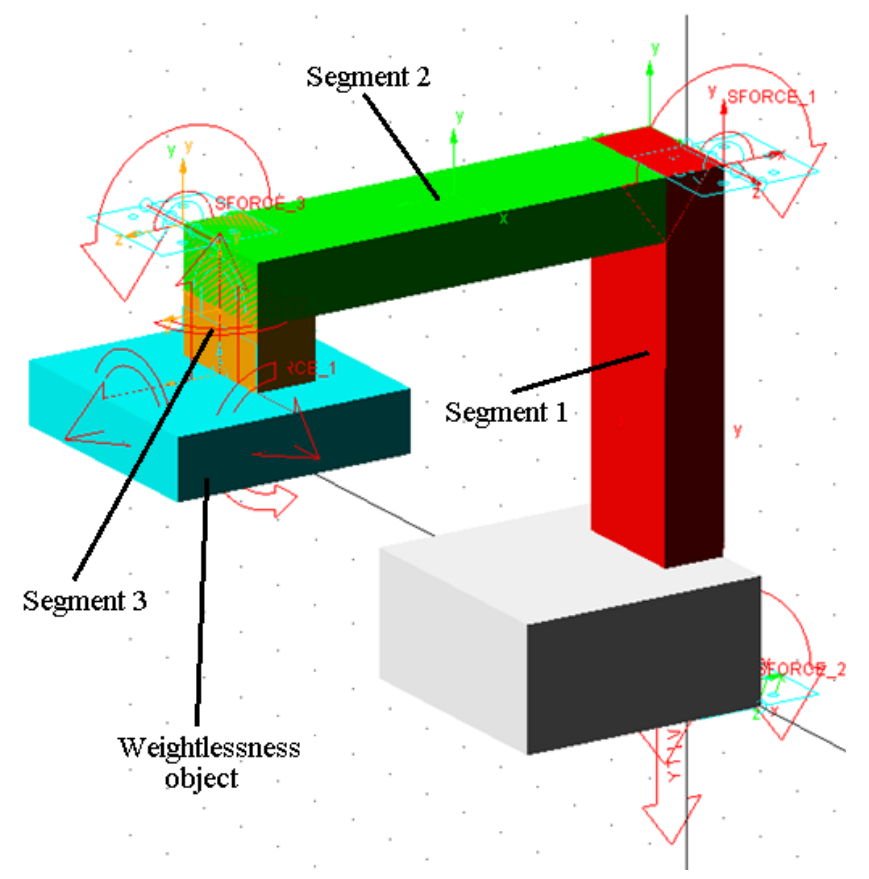

Fig. 4. Model of the manipulator arm with 3 degrees of freedom

For checking the obtained solution the model was designed in solid modeling system (figure 4). It represents key system parameters and necessary environment: gravity force, mass and inertia characteristics of arm segments and load, torque interplay between arm segments. Elaborated model reproduce minimal essential physical interactions for equations system (3) accuracy check.

Another model where weightlessness manipulator arm and gravity force were removed was designed for control. The model has to represent object behavior in weightlessness with same force impact as in the model with manipulator arm. If elaborated model is accurate, movement characteristics should coincide in both cases.

In the course of operation arbitrary force and torque were exerted to the weightlessness object. Comparison of both results shows some difference in models behavior. The difference is presence of oscillating processes in test bench model with arm manipulator. The sources of oscillating processes are discrete computational methods of modeling and inertialess sensors model.

In the models comparison contrary direction forces and force moments were applied sequentially (figure 5). It's necessary to stop load movement avoiding its infinite motion.

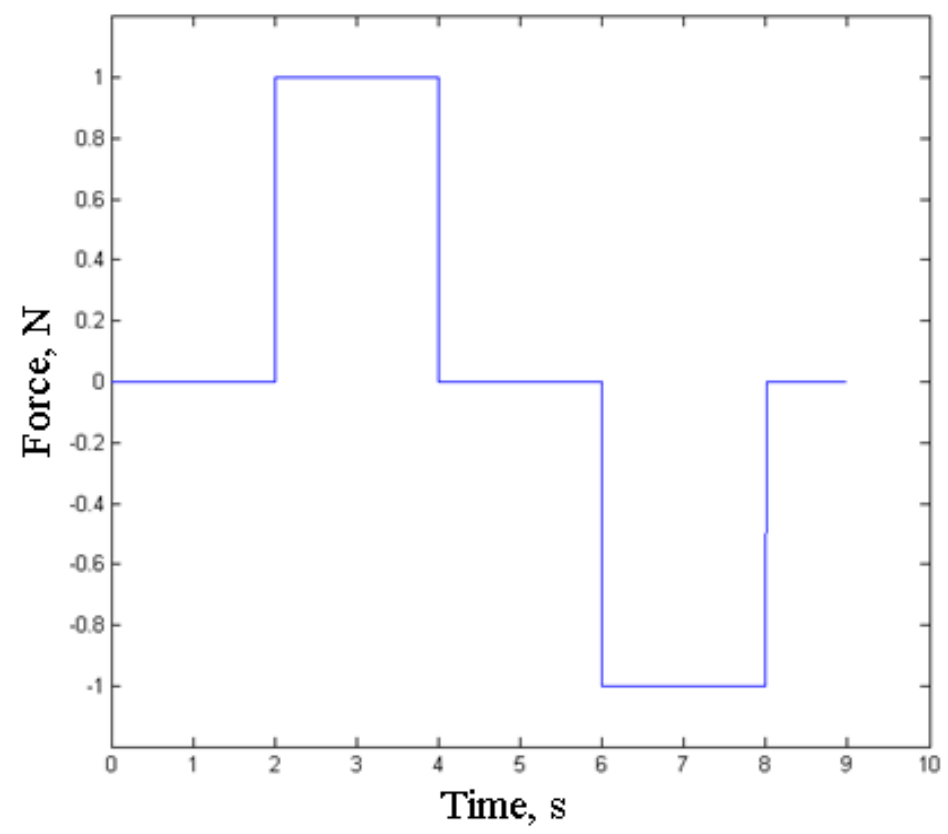

Fig. 5. Force to time graph 
Comparison of modeling results of weightlessness test bench and zero gravity models.

\begin{tabular}{llcc}
\hline \multicolumn{1}{c}{ Impact } & \multicolumn{1}{c}{ Expected result } & $\begin{array}{c}\text { Modeling result meets the } \\
\text { expectation? }\end{array}$ \\
\cline { 2 - 4 } & \multicolumn{1}{c}{$\begin{array}{c}\text { Test bench } \\
\text { model }\end{array}$} & $\begin{array}{c}\text { Zero gravity } \\
\text { model }\end{array}$ \\
\hline Y-axis force & $\begin{array}{l}\text { Load acceleration along } \\
\text { Y-axix, then its } \\
\text { stopping }\end{array}$ & + & + \\
X-axis torque & $\begin{array}{l}\text { Load rotation around } \\
\text { mass center without } \\
\text { location changing } \\
\text { plain movement of } \\
\text { complex load }\end{array}$ & + & + \\
$\begin{array}{l}\text { Y-axis force and } \\
\text { torque around X- } \\
\text { axis } \\
\text { No external forces }\end{array}$ & Load is motionless & + & + \\
\hline
\end{tabular}

Table 1. Modeling results comparison

With table 1 it's clear that in most cases modeling results match. Only difference appears with long time load motionless holding. Depending on modeling sample time and system parameters the manipulator arm load starts to oscillate in different period. At the present stage it's assumed that oscillation processes are insignificant, because with more accurate model including joints friction and sensors inertia oscillation amplitude will become inconspicuous.

\section{Conclusion}

The initial step of work on developing of weightlessness test bench model was carried out. Preparatory control system was elaborated and tuned. Control system solves weightlessness simulation problem for constant geometry load, held with three degrees of freedom manipulator arm.

Future key lines of work are:

- model of the holding manipulator arm expansion to six degrees of freedom and operated object free displacing;

- transition from torque control to acceleration control of joints;

- $\quad$ six component force and torque sensor addition in the model. Performance with sensor values taken into account;

- model behavior exploration with sensor non-ideality, measurement and data calculation accuracy consideration. Addition sensors discretization to the model, measure delay time and noise addition etc.

In process of listed problems solving the test bench control system for space robotic systems weightlessness test will be elaborated. Estimated system parameters (sensor accuracy, data processing rate, manipulator arm responsiveness and other) will be defined. Also test bench possibilities for different applicative problems will be explored.

The applied scientific investigations are carried out with the financial support of the Russian Federation represented by the Russian Ministry of Education and Science. The unique identifier of the Applied scientific investigations is RFMEFI57814X0046.

\section{References}

[1] Ahmet Shala, Rame Likaj, Mirlind Bruqi, Xhevahir Bajrami. Propulsion Effect Analysis of 3DoF Robot under Gravity. Procedia Engineering 100 (2015), pp. 206-212.

[2] Victor Titov, Igor Shardyko, Sergey Isaenko. Force-Torque Control Implementation for 2 DoF Manipulator. Procedia Engineering 69 (2014), pp. 1232-1241.

[3] Sebastian Schwarz, Mathias v. Alberti. Sentinel-1 SAR Deployment Testing - Lessons Learned. Proceedings of ESMATS 2015, 16th European Space Mechanisms and Tribology Symposium.

[4] A.N.Timofeev, I.V.Shardyko. Problems of anthropomorphic robot application in space. Robotics and Technical Cybernetics (Робототехника и техническая кибернетика), 1(1) 2013. pp. 37-41.

[5] V.A. Lopota, A.M. Potapov. Manipulation system for extravehicular activity support. Mechatronics, automatization and control (Мехатроника, автоматизация и управление), 2011. №2, pp. 6-16. ISSN 1684-6427. 
[6] A.I. Kargov, A.A. Gradovtsev. Design of space transport and manipulation system for work on ISS. Extremal robotics conference papers (Экстремальная робототехника, труды конференции), 2010, pp. 97-103. ISBN 9785-904031-71-8.

[7] I.U. Dalyaev. Space transport and manipulation system for servicing of Russian Orbital Segment of ISS. Bauman MSTU Vestnik (Вестник МГТУ им. Н.Э. Баумана), 2011. 